\title{
ASYMPTOTIC EXPANSIONS OF TRACES FOR CERTAIN CONVOLUTION OPERATORS
}

BY

\author{
RAYMOND ROCCAFORTE
}

\begin{abstract}
A version of Szegö's theorem in Euclidean space gives the first two terms of the asymptotics as $\alpha \rightarrow \infty$ of the determinant of convolution operators on $L_{2}(\alpha \Omega)$, where $\Omega$ is a bounded subset of $\mathbf{R}^{n}$ with smooth boundary. In this paper the more general problem of the asymptotics of traces of certain analytic functions of the operators is considered and the next term in the expansion is obtained.
\end{abstract}

1. Introduction. In $1960 \mathrm{H}$. Widom [6] discovered the following analogue of theorems of G. Szegö [2, §5.5] and M. Kac [3]. Consider the operator

$$
T_{\alpha}: f \rightarrow \int_{\alpha \Omega} k(x-y) f(y) d y
$$

on $L_{2}(\alpha \Omega)$, where $\Omega$ is a compact subset of $\mathbf{R}^{n}$ with $C^{1}$ boundary and $\alpha$ is a real parameter. With

$$
\begin{gathered}
\sigma(\xi)=\hat{k}(\xi)=\int_{\mathbf{R}^{n}} k(z) e^{-i \xi \cdot z} d z \\
g^{\vee}(z)=\frac{1}{(2 \pi)^{n}} \int_{\mathbf{R}^{n}} g(\xi) e^{i \xi \cdot z} d \xi \quad \text { and } \quad s(z)=(\log (1+\sigma))^{\vee}(z)
\end{gathered}
$$

Widom proved that under suitable conditions

$$
\log \operatorname{det}\left(1+T_{\alpha}\right)=a_{0} \alpha^{n}+a_{1} \alpha^{n-1}+o\left(\alpha^{n-1}\right), \quad \alpha \rightarrow \infty,
$$

where

$$
a_{0}=\operatorname{vol}(\Omega) s(0), \quad a_{1}=\frac{1}{4} \int_{\partial \Omega} \int_{\mathbf{R}^{n}}\left|z \cdot n_{x}\right| s(z) s(-z) d z d A .
$$

Here $n_{x}$ is the inward pointing unit normal at $x \in \partial \Omega$ and $d A$ is surface measure on $\partial \Omega$. In [7] Widom found a generalization of this in the case $k$ is matrix valued and $L_{2}(\alpha \Omega)$ consists of vector valued functions.

In this paper the following extension is considered. If $F$ is a function analytic on the spectrum of $T_{\alpha}$, then $F\left(T_{\alpha}\right)$ is defined and will be trace class if $F(0)=0$. It is shown here that in the scalar case there is an expansion

$$
\operatorname{tr} F\left(T_{\alpha}\right)=a_{0} \alpha^{n}+a_{1} \alpha^{n-1}+a_{2} \alpha^{n-2}+o\left(\alpha^{n-2}\right), \quad \alpha \rightarrow \infty,
$$

Received by the editors April 25, 1983 and, in revised form, August 23, 1983.

1980 Mathematics Subject Classification. Primary 47B10, 47B35; Secondary 45E10.

Key words and phrases. Szegö limit theorem, convolution operators, Wiener-Hopf operators, asymptotics of traces. 
where $a_{0}$ and $a_{1}$ are given by (1.2) in the case $F$ is the logarithm and the evaluation of $a_{2}$ is new. In the matrix case an expression is obtained only when $F$ is a power, i.e., $F(\lambda)=\lambda^{m}$ for some positive integer $m$. The coefficients $a_{0}$ and $a_{1}$ agree with those given in [7] and again the evaluation of $a_{2}$ is new.

The assumptions and notation will be as follows. Let $k \in L_{1}\left(\mathbf{R}^{n}\right)$ and suppose $\sigma=\hat{k}$ is also in $L_{1}\left(\mathbf{R}^{n}\right)$. In addition, it is assumed that

$$
\int_{\mathbf{R}^{n}}|x|^{2}|k(x)| d x<\infty
$$

where, if $k$ is matrix valued, $|k(x)|$ denotes the Hilbert-Schmidt norm of $k(x)$.

$\Omega$ will be a compact subset of $\mathbf{R}^{n}(n>1)$ whose boundary is a $C^{3}$ hypersurface. $T^{*}(\partial \Omega)$ will denote the cotangent bundle of $\partial \Omega$ which is identified with the tangent bundle $T(\partial \Omega)$ via the Riemannian metric on $\partial \Omega\left(\mathbf{R}^{n}\right.$ inner product restricted to tangent spaces). This induces a natural measure $d \bar{\xi} d A$ on $T^{*}(\partial \Omega)$, where $d A$ is surface measure on $\partial \Omega$ and $d \bar{\xi}$ is Lebesgue measure on the hyperplane $T_{x}(\partial \Omega)$. The notation $\xi=(\bar{\xi}, \eta)=\left(\xi^{1}, \ldots, \xi^{n-1}, \eta\right)$ will be used for vectors in $T_{x}(\partial \Omega) \times \mathbf{R}$, the last coordinate $\eta$ being with respect to the unit inward normal to $\partial \Omega$ at $x$. Superscripts $i, j=1, \ldots, n-1$ on a function will denote differentiation in the tangential direction (i.e., $\left.\tau^{i}=\partial \tau / \partial \bar{\xi}^{i}\right)$ and $\operatorname{grad}_{\xi}(\tau)$ will mean $\left(\tau^{1}, \ldots, \tau^{n-1}\right)$. The superscript $n$ will denote differentiation in the normal direction.

Finally, $L$ will denote the second fundamental form for $\partial \Omega$ with respect to the inward unit normal and $H$ will denote $n-1$ times the mean curvature of $\partial \Omega$. The main result in the scalar case can now be stated.

THEOREM 1.1. Let $F$ be analytic on a disc of radius greater than $\|k\|_{1}$ and suppose $F(0)=0$. Then under the above assumptions

where

$$
\operatorname{tr} F\left(T_{\alpha}\right)=a_{0} \alpha^{n}+a_{1} \alpha^{n-1}+a_{2} \alpha^{n-2}+o\left(\alpha^{n-2}\right), \quad \alpha \rightarrow \infty,
$$

$$
\begin{aligned}
a_{0} & =\operatorname{vol}(\Omega) \frac{1}{(2 \pi)^{n}} \int F(\sigma(\xi)) d \xi, \\
a_{1} & =\frac{1}{4} \frac{1}{(2 \pi)^{n}} \int_{T^{*}(\partial \Omega) \times \mathbf{R}} \int \frac{F(\sigma(\eta))-F(\sigma(\zeta))}{\sigma(\eta)-\sigma(\zeta)} \frac{\sigma^{n}(\zeta)-\sigma^{n}(\eta)}{\zeta-\eta} d \zeta d \xi d A, \\
a_{2}= & -\frac{1}{8 \pi^{2}} \frac{1}{(2 \pi)^{n}} \int_{T^{*}(\partial \Omega) \times \mathbf{R}} \iint\left\{\sum_{a \in A_{3}} \frac{F\left(\sigma\left(\eta^{a}\right)\right)}{\left(\sigma\left(\eta^{a}\right)-\sigma\left(\zeta_{1}^{a}\right)\right)\left(\sigma\left(\eta^{a}\right)-\sigma\left(\zeta_{2}^{a}\right)\right)}\right\} \\
& \times\left\{L\left(\operatorname{grad}_{\xi} \sigma\left(\zeta_{1}\right), \operatorname{grad}_{\xi} \sigma\left(\zeta_{2}\right)\right)-H \cdot \sigma^{n}\left(\zeta_{1}\right) \sigma^{n}\left(\zeta_{2}\right)\right\} \frac{d \zeta_{1}}{\zeta_{1}-\eta} \frac{d \zeta_{2}}{\zeta_{2}-\eta} d \xi d A .
\end{aligned}
$$

Here $A_{3}$ is the alternating group on the 3 symbols $\eta, \zeta_{1}, \zeta_{2}$, and expressions like $\sigma\left(\zeta_{1}\right)$ mean $\sigma\left(\xi^{1}, \ldots, \xi^{n-1}, \zeta_{1}\right)$.

The proof proceeds as follows. The kernel of $T_{\alpha}^{m}$ at a point $(x, y) \in \alpha \Omega \times \alpha \Omega$ is

$$
\begin{gathered}
\int \cdots \int k\left(x-x_{1}\right) k\left(x_{1}-x_{2}\right) \cdots k\left(x_{m-2}-x_{m-1}\right) k\left(x_{m-1}-y\right) \\
\quad \times \chi_{\alpha \Omega}\left(x_{1}\right) \cdots \chi_{\alpha \Omega}\left(x_{m-1}\right) d x_{1} \cdots d x_{m-1},
\end{gathered}
$$


where the integration is taken over $\mathbf{R}^{n} \times \cdots \times \mathbf{R}^{n}$ and where $\chi_{S}$ denotes the characteristic function of the set $S$. Change variables: let $\bar{x}_{1}=x-x_{1}, \bar{x}_{2}=x_{1}-$ $x_{2}, \ldots, \bar{x}_{m-1}=x_{m-2}-x_{m-1}$. This gives

$$
\begin{aligned}
& \int \cdots \int k\left(x_{1}\right) \cdots k\left(x_{m-1}\right) k\left(x-y-x_{1}-\cdots-x_{m-1}\right) \chi_{\alpha \Omega}\left(x-x_{1}\right) \\
& \quad \times \chi_{\alpha \Omega}\left(x-x_{1}-x_{2}\right) \cdots \chi_{\alpha \Omega}\left(x-x_{1}-\cdots-x_{m-1}\right) d x_{1} \cdots d x_{m-1} .
\end{aligned}
$$

Under the above hypotheses this expression is continuous for $m \geqslant 2$ (the case $m=1$ is easily handled; see [3, pp. 506-507]) so to find $\operatorname{tr} T_{\alpha}^{m}$ the trace of the kernel is integrated over the diagonal of $\alpha \Omega \times \alpha \Omega$. The result is

$$
\begin{aligned}
& \operatorname{tr}\left(T_{\alpha}^{m}\right)=\int \cdots \int \operatorname{tr} k\left(x_{1}\right) \cdots k\left(x_{m-1}\right) k\left(-x_{1}-\cdots-x_{m-1}\right) \\
& \quad \times \operatorname{vol}\left(\alpha \Omega \cap\left(\alpha \Omega+x_{1}\right) \cap \cdots \cap\left(\alpha \Omega+x_{1}+\cdots+x_{m-1}\right)\right) d x_{1} \cdots d x_{m-1} .
\end{aligned}
$$

In $\S 2$ an expression is obtained for the volume in the integrand and in $\S 3$ this is used to derive a formula for $\operatorname{tr}\left(T_{\alpha}^{m}\right)$. The latter and an identity of F. Spitzer (see [1]) are then used in $\$ 4$ to prove Theorem 1.1.

If $\|k\|_{1}<1$ then $\log (1+\lambda)$ satisfies the hypotheses of Theorem 1.1 and there results an expansion for $\operatorname{tr} \log \left(1+T_{\alpha}\right)$. It is not immediate that the $a_{1}$ term of this expansion is the same as the $a_{1}$ term appearing in (1.2). In $\$ 5$ this is shown to be the case. An argument similar to but more complicated than the one in $\$ 5$ also shows that the expression for $a_{2}$ in Theorem 1.1 is equivalent, in the case $F(\lambda)=\log (1+\lambda)$, to one recently derived by Widom [8].

ACKNOWLEDGEMENT. It is the author's pleasure to thank Harold Widom for suggesting this problem and for his subsequent advice and encouragement.

2. The volume estimate. In obtaining an expression for the volume in the integrand of (1.4) it is convenient to divide by $\alpha^{n}$ and set $\varepsilon=\alpha^{-1}$.

THEOREM 2.1. Let $\Omega$ be a compact subset of $\mathbf{R}^{n}(n>1)$ whose boundary is a $C^{3}$ hypersurface. Let $v_{1}, \ldots, v_{r} \in \mathbf{R}^{n}$ and define $\Omega_{\varepsilon}=\Omega \cap\left(\Omega+\varepsilon v_{1}\right) \cap \cdots \cap\left(\Omega+\varepsilon v_{r}\right)$. Then

$$
\begin{aligned}
\operatorname{vol}\left(\Omega \backslash \Omega_{\varepsilon}\right)= & \int_{\partial \Omega} \max _{i=1, \ldots, r}\left\{0, \varepsilon v_{i} \cdot n_{x}+\frac{\varepsilon^{2}}{2} L\left(v_{i_{t}}, v_{i_{t}}\right)\right\} d A(x) \\
& -\frac{\varepsilon^{2}}{2} \int_{\partial \Omega}\left(\max _{i=1, \ldots, r}\left\{0, v_{i} \cdot n_{x}\right\}\right)^{2} H d A(x)+o\left(\varepsilon^{2}\right), \quad \varepsilon \rightarrow 0,
\end{aligned}
$$

where $n_{x}$ is the unit inward normal at $x \in \partial \Omega, L$ and $H$ are as in the introduction, and $w_{t}$ is the component of $w$ tangent to $\partial \Omega$ at $x$.

Proof. Since $\partial \Omega$ is compact there exists an $\varepsilon_{0}$ and an $\varepsilon_{0}$-tubular neighborhood $N_{0}$ of $\partial \Omega$ such that each $x \in N_{0}$ can be written uniquely as $x=\bar{x}+s n_{\bar{x}}$, where $\bar{x} \in \partial \Omega$ and $|s|<\varepsilon_{0}$. If $\varepsilon$ is small enough then $\Omega \backslash \Omega_{\varepsilon} \subset N_{0}$. 
Let $\left\{C_{\tau}, \psi_{\tau}\right\}$ be an atlas of coordinate neighborhoods covering $\partial \Omega$ and pick a finite subcover. Let $D_{\tau}=\left\{x \in N_{0}\right.$ : if $x=\bar{x}+s n_{\bar{x}}$, then $\left.\bar{x} \in C_{\tau}\right\}$. Define $\phi_{\tau}: D_{\tau} \rightarrow$ $\mathbf{R}^{n-1} \times \mathbf{R}$ as follows: if $x=\bar{x}+s n_{\bar{x}} \in D_{\tau}$ and $\psi_{\tau}(\bar{x})=\bar{u} \in \mathbf{R}^{n-1}$, then $\phi_{\tau}: \bar{x}+s n_{\bar{x}}$ $\rightarrow \bar{u}+s \nu$, where $\nu$ is the unit vector in $\mathbf{R}^{n-1} \times \mathbf{R}$ normal to $\mathbf{R}^{n-1}$. By the compactness of $\Omega$ there exist open sets $N_{\tau} \subset D_{\tau}$ such that the $N_{\tau}$ are an open cover of $N_{0}$ and the distance from $N_{\tau}$ to the complement of $D_{\tau}$ is, for all $\tau$, greater than some $\varepsilon_{1}$. If $\varepsilon$ is chosen such that $\max \left|\varepsilon v_{i}\right|<\varepsilon_{1}$ then, for all $\tau, x \in N_{\tau}$ implies $x-\varepsilon v_{i} \in D_{\tau}$. Let $B_{\tau}=N_{\tau} \cap \partial \Omega \subset C_{\tau}$.

Let $\left\{\rho_{\tau}\right\}$ be a partition of unity subordinate to the cover $\cup B_{\tau}$ of $\partial \Omega$. Each $\rho_{\tau}$ extends to a function $\tilde{\rho}_{\tau}$ on $N_{\tau} \cap \Omega \backslash \Omega_{\varepsilon}$ by defining $\tilde{\rho}_{\tau}\left(\bar{x}+s n_{\bar{x}}\right)=\rho_{\tau}(\bar{x})$. It now suffices to prove

$$
\begin{aligned}
\int_{N_{\tau} \cap \Omega \backslash \Omega_{\varepsilon}} \tilde{\boldsymbol{\rho}}_{\tau} d V= & \int_{B_{\tau}} \boldsymbol{\rho}_{\tau}(\bar{x}) \max _{i}\left\{0, \varepsilon v_{i} \cdot n_{\bar{x}}+\frac{\varepsilon^{2}}{2} L\left(v_{i_{t}}, v_{i_{t}}\right)\right\} d A \\
& -\frac{\varepsilon^{2}}{2} \int_{B_{\tau}} \rho_{\tau}(\bar{x})\left(\max _{i}\left\{0, v_{i} \cdot n_{\bar{x}}\right\}\right)^{2} H d A+o\left(\varepsilon^{2}\right),
\end{aligned}
$$

where $d V$ denotes the volume element on $\mathbf{R}^{n}$.

In what follows the index $\tau$ will be dropped. From the construction of $\phi$ it follows that for $y \in D, y \in \Omega \cap D$ if and only if $\phi(y) \cdot \nu=s \geqslant 0$. Hence for $x \in N$,

$$
x \in \Omega_{\varepsilon} \cap N=N \cap \Omega \cap\left(\Omega+\varepsilon v_{1}\right) \cap \cdots \cap\left(\Omega+\varepsilon v_{r}\right)
$$

if and only if $s \geqslant 0$ and, for all $i, \phi\left(x-\varepsilon v_{i}\right) \cdot \nu \geqslant 0$. By Taylor's theorem $x \in N \cap \Omega_{\varepsilon}$ if and only if $s \geqslant 0$ and, for all $i$,

$$
\phi(x) \cdot \nu-\varepsilon d \phi_{x}\left(v_{i}\right) \cdot \nu+\frac{\varepsilon^{2}}{2} d^{2} \phi_{x}\left(v_{i}, v_{i}\right) \cdot \nu+R(\varepsilon) \geqslant 0,
$$

where $R(\varepsilon)=o\left(\varepsilon^{2}\right)$, the estimate being uniform over $x$ since $d^{2} \phi$ is continuous.

Lemma 2.2. For all $w \in \mathbf{R}^{n}$ and all $x=\bar{x}+s n_{\bar{x}} \in N$

(i) $d \phi_{x}(w) \cdot \nu=w \cdot n_{\bar{x}}$

(ii) $d^{2} \phi_{x}(w, w) \cdot \nu=-L\left(w_{t}, w_{t}\right)+r(s)$,

where $\lim _{s \rightarrow 0} r(s)=0$ uniformly in $x$ and $L$ is evaluated at $\bar{x}$.

The proof will appear later in the section. From (2.2) and the first part of the lemma, $x \in N \cap \Omega \backslash \Omega_{\varepsilon}$ if and only if

$$
\begin{gathered}
s \geqslant 0 \text { and, for some } i, \\
s-\varepsilon v_{i} \cdot n_{\bar{x}}+\frac{\varepsilon^{2}}{2} d^{2} \phi_{x}\left(v_{i}, v_{i}\right) \cdot \nu+R(\varepsilon)<0 .
\end{gathered}
$$

By part (ii) of the lemma, $x \in N \cap \Omega \backslash \Omega_{\varepsilon}$ if and only if $s \geqslant 0$ and, for some $i$,

$$
s-\varepsilon v_{i} \cdot n_{\bar{x}}-\frac{\varepsilon^{2}}{2} L\left(v_{i_{t}}, v_{i_{t}}\right)+R_{1}(\varepsilon)<0,
$$

where $R_{1}(\varepsilon)=\varepsilon^{2} r(s) / 2+R(\varepsilon)=o\left(\varepsilon^{2}\right)$ (since (2.3) implies $s \rightarrow 0$ as $\varepsilon \rightarrow 0$ ). It follows that

$$
N \cap \Omega \backslash \Omega_{\varepsilon}=N \cap \Omega \backslash S_{\varepsilon},
$$


where $S_{\varepsilon}=\left\{x \in N: s \geqslant 0\right.$ and, for all $\left.i, s-\varepsilon v_{i} \cdot n_{\bar{x}}-\varepsilon^{2} L\left(v_{i_{i}}, v_{i_{t}}\right) / 2+R(\varepsilon) \geqslant 0\right\}$. Define $I_{0}=\left\{x \in N: s \geqslant 0\right.$ and, for all $\left.i, s-\varepsilon v_{i} \cdot n_{\bar{x}}-\varepsilon^{2} L\left(v_{i_{t}}, v_{i_{t}}\right) / 2 \geqslant 0\right\}$.

LEMMA 2.3. $\operatorname{vol}\left(S_{\varepsilon} \Delta I_{0}\right)=o\left(\varepsilon^{2}\right), \varepsilon \rightarrow 0$.

Again the proof is deferred to later in the section. From (2.4) and Lemma 2.3 it follows that $N \cap \Omega \backslash \Omega_{\varepsilon}$ can be replaced by $N \cap \Omega \backslash I_{0}$ in (2.1).

By the change of variables formula,

$$
\begin{aligned}
& \int_{\Omega \cap N \backslash I_{0}} \tilde{\rho} d V=\int_{\phi\left(\Omega \cap N \backslash I_{0}\right)}\left(\tilde{\rho} \circ \phi^{-1}\right)\left|d \phi^{-1}{ }_{u}\right| d u^{1} \cdots d u^{n-1} d s \\
& \quad=\int_{\phi\left(\Omega \cap N \backslash I_{0}\right)}\left(\tilde{\rho} \circ \phi^{-1}\right)\left\{\left|d \phi^{-1}\right|+d\left(\left|d \phi^{-1}{ }_{(\cdot)}\right|\right)_{\bar{u}}(s \nu)+o(s)\right\} d u^{1} \cdots d u^{n-1} d s,
\end{aligned}
$$

where by the definitions of $\phi$ and $I_{0}$

$$
\phi\left(\Omega \cap N \backslash I_{0}\right)=\left\{u \in \phi(N): 0 \leqslant s<\max _{i}\left(0, \varepsilon v_{i} \cdot n_{\bar{x}}+\frac{\varepsilon^{2}}{2} L\left(v_{i_{t}}, v_{i_{t}}\right)\right)\right\} .
$$

Integrating the $o(s)$ term within the indicated limits yields something which is $o\left(\varepsilon^{2}\right)$. Since $\tilde{\rho}$ is constant with respect to $s$, integrating the first term of $(2.5)$ with respect to $s$ yields

$$
\int_{\phi(B)}\left(\rho \circ \phi^{-1}\right) \max _{i}\left\{0, \varepsilon v_{i} \cdot n_{\bar{x}}+\frac{\varepsilon^{2}}{2} L\left(v_{i_{t}}, v_{i_{t}}\right)\right\}\left|d \phi_{\bar{u}}^{-1}\right| d u^{1} \cdots d u^{n-1}
$$

which equals the first term on the right side of (2.1).

Integrating the remaining term in (2.5) with respect to $s$ yields

$$
\frac{\varepsilon^{2}}{2} \int_{\phi(B)}\left(\rho \circ \phi^{-1}\right)\left(\max \left(0, v_{i} \cdot n_{\bar{x}}\right)\right)^{2} d\left(\mid d \phi^{-1}(\cdot)\right)_{\bar{u}}(\nu) d u^{1} \cdots d u^{n-1}+o\left(\varepsilon^{2}\right) .
$$

By a classical result from surface theory $[5, \mathrm{p} .159]$ this last integral equals

$$
-\frac{\varepsilon^{2}}{2} \int_{B} \rho\left(\max \left(0, v_{i} \cdot n_{x}\right)\right)^{2} H d A .
$$

Comparison with (2.1) shows the theorem is proved.

COROLlaRY 2.4. Except for a set of $\left(v_{1}, \ldots, v_{r}\right)$ of measure zero in $\mathbf{R}^{n} \times \cdots \times \mathbf{R}^{n}$

$$
\begin{aligned}
\operatorname{vol}\left(\Omega \backslash \Omega_{\varepsilon}\right)= & \varepsilon \int_{\partial \Omega i=1, \ldots, r}\left\{0, v_{i} \cdot n_{x}\right\} d A(x) \\
& +\frac{\varepsilon^{2}}{2} \sum_{q=1}^{r} \int_{S_{q}} L\left(v_{q_{t}}, v_{q_{t}}\right)-\left(v_{q} \cdot n_{x}\right)^{2} H d A(x)+o\left(\varepsilon^{2}\right), \quad \varepsilon \rightarrow 0,
\end{aligned}
$$

where $S_{q}=\left\{x \in \partial \Omega: v_{q} \cdot n_{x}=\max _{i}\left(0, v_{i} \cdot n_{x}\right)\right\}$.

Proof. For fixed $x \in \partial \Omega$ and fixed $v \in \mathbf{R}^{n}$, the set of $w \in \mathbf{R}^{n}$ such that $v \cdot n_{x}=w$ - $n_{x}$ is a hyperplane and so has measure zero in $\mathbf{R}^{n}$. Thus the set of $\left(v_{1}, v_{2}\right) \in \mathbf{R}^{n} \times \mathbf{R}^{n}$ 
such that $v_{1} \cdot n_{x}=v_{2} \cdot n_{x}$ has measure zero and it follows that the set of $\left(v_{1}, \ldots, v_{r}\right)$ $\in \mathbf{R}^{n} \times \cdots \times \mathbf{R}^{n}$ such that $\max _{i}\left(0, v_{i} \cdot n_{x}\right)$ occurs at more than one of the $v_{i}$ has measure zero in $\mathbf{R}^{n} \times \cdots \times \mathbf{R}^{n}$.

Thus the set $E=\left\{\left(x, v_{1}, \ldots, v_{r}\right) \in \partial \Omega \times \mathbf{R}^{n} \times \cdots \times \mathbf{R}^{n}: \max \left(0, v_{i} \cdot n_{x}\right)\right.$ occurs at more than one of the $\left.v_{i}\right\}$ has measure zero in $\partial \Omega \times \mathbf{R}^{n} \times \cdots \times \mathbf{R}^{n}$. By Fubini's theorem, for almost every $\left(v_{1}, \ldots, v_{r}\right) \in \mathbf{R}^{n} \times \cdots \times \mathbf{R}^{n}$ the set $E_{\left(v_{1}, \ldots, v_{r}\right)}=\{x \in \partial \Omega$ : $\left.\left(x, v_{1}, \ldots, v_{r}\right) \in E\right\}$ has measure zero in $\partial \Omega$. Thus for almost every $\left(v_{1}, \ldots, v_{r}\right)$ the sets $S_{q}=\left\{x \in \partial \Omega: v_{q} \cdot n_{x}>\max _{q \neq 1}\left(0, v_{i} \cdot n_{x}\right)\right\}, q=1, \ldots, r$, and $S_{0}=\{x \in \partial \Omega$ : $\left.\max _{i}\left(0, v_{i} \cdot n_{x}\right)=0\right\}$ form a disjoint cover of $\partial \Omega$ except for a set of measure zero. Note that on $S_{q}, q=1, \ldots, r$, the error obtained by replacing the integrand

$$
\max _{i}\left(0, \varepsilon v_{i} \cdot n_{x}+\frac{\varepsilon^{2}}{2} L\left(v_{i_{t}}, v_{i_{t}}\right)\right)
$$

in Theorem 2.1 by $\varepsilon v_{q} \cdot n_{x}+\varepsilon^{2} L\left(v_{q_{t}}, v_{q_{t}}\right) / 2$ is $O\left(\varepsilon^{2}\right)$ and likewise for replacing the integrand with 0 on $S_{0}$.

For $\delta>0$, define $S_{q \delta}=\left\{x \in \partial \Omega: v_{q} \cdot n_{x}>\max _{i \neq q}\left(0, v_{i} \cdot n_{x}\right)+\delta\right\}$ for $q=$ $1, \ldots, r$ and $S_{0 \delta}=\left\{x \in \partial \Omega: \max _{i}\left(v_{i} \cdot n_{x}\right)<-\delta\right\}$. Then $S_{q \delta} \subset S_{q}$ for $q=0,1, \ldots, r$ and on each $S_{q \delta}$ there is an $\varepsilon_{q \delta}$ such that for $\varepsilon<\varepsilon_{q \delta}$ no error results from the replacements indicated above.

Hence for $\varepsilon<\varepsilon_{q \delta}$ the total error on $S_{q}, q=0,1, \ldots, r$, is less than

$$
D_{q} \cdot \operatorname{meas}\left(S_{q} \backslash S_{q \delta}\right) \varepsilon^{2}
$$

for some constant $D_{q}$. Since meas $\left(S_{q} \backslash S_{q \delta}\right)$ can be made arbitrarily small by choosing $\delta$ small enough, the error is $o\left(\varepsilon^{2}\right)$.

The remainder of the section consists of the proofs of Lemmas 2.2 and 2.3 followed by another lemma which will be useful later.

Proof OF LEMMA 2.2. To prove part (i) note that since

$$
d \phi_{x}(w) \cdot \nu=d \phi_{x}\left(w_{t}\right) \cdot \nu+d \phi_{x}\left(\left(w \cdot n_{\bar{x}}\right) n_{\bar{x}}\right) \cdot \nu
$$

it suffices to prove

$$
d \phi_{x}\left(n_{\bar{x}}\right)=\nu \text { and } d \phi_{x}\left(w_{t}\right) \cdot \nu=0 \text {. }
$$

For each fixed $s_{0}$ the map $\phi^{-1}:\left(\bar{u}, s_{0}\right) \rightarrow \bar{x}(\bar{u})+s_{0} n_{\bar{x}}(\bar{u})$ describes the hypersurface $B+s_{0} n_{\bar{x}}$. The vectors $\partial\left(\bar{x}+s_{0} n_{\bar{x}}\right) /\left.\partial u^{i}\right|_{\bar{u}_{0}}$ form a basis for the tangent space to $B+s_{0} n_{\bar{x}}$ at $\bar{x}\left(\bar{u}_{0}\right)+s_{0} n_{\bar{x}}\left(\bar{u}_{0}\right)$. Thus $d \phi_{\left(\bar{u}_{0}, s_{0}\right)}^{-1}$ sends vectors $(\bar{u}, 0)$ to vectors tangent to $B+s_{0} n_{\bar{x}}$ at $\bar{x}\left(\bar{u}_{0}\right)+s_{0} n_{\bar{x}}\left(\bar{u}_{0}\right)$ and sends $\nu=(0, \ldots, 0,1)$ to $n_{\bar{x}}$. Hence it suffices to show that for any $x=\bar{x}+s n_{\bar{x}}, T_{x}\left(B+s n_{\bar{x}}\right)=T_{\bar{x}}(\partial \Omega)$. But

$$
n_{\bar{x}} \cdot \frac{\partial}{\partial u^{i}}\left(\bar{x}+s n_{\bar{x}}\right)=s n_{\bar{x}} \cdot \frac{\partial}{\partial u^{i}} n_{\bar{x}}=\frac{1}{2} s \frac{\partial}{\partial u^{i}}\left(n_{\bar{x}} \cdot n_{\bar{x}}\right)=0,
$$

so the tangent space $T_{x}\left(B+s n_{\bar{x}}\right)$ is normal to $n_{\bar{x}}$ and hence the same as $T_{\bar{x}}(\partial \Omega)$.

Now the proof of part (ii). Since $d^{2} \phi$ is uniformly continuous on $N$,

$$
d^{2} \phi_{x}(w, w) \cdot \nu=d^{2} \phi_{\bar{x}}(w, w) \cdot \nu+r(s)
$$


where $\lim _{s \rightarrow 0} r(s)=0$ uniformly in $x$. Also, since

$$
\begin{aligned}
d^{2} \phi_{x}\left(w, n_{\bar{x}}\right) \cdot \nu & =\left(\lim _{s \rightarrow 0} \frac{d \phi_{x+s n_{\bar{x}}}(w)-d \phi_{x}(w)}{s}\right) \cdot \nu \\
& =\lim _{s \rightarrow 0} \frac{w \cdot n_{\bar{x}}-w \cdot n_{\bar{x}}}{s} \quad(\text { by Lemma 2.2) } \\
& =0, \\
d^{2} \phi_{\bar{x}} & (w, w) \cdot \nu=d^{2} \phi_{\bar{x}}\left(w_{t}, w_{t}\right) \cdot \nu .
\end{aligned}
$$

Hence it suffices to show

$$
d^{2} \phi_{\bar{x}}\left(w_{t}, w_{t}\right) \cdot \nu=-L\left(w_{t}, w_{t}\right) .
$$

Fix $\bar{x} \in B$. It suffices to assume $\bar{x}=0 \in \mathbf{R}^{n}$ and that $\psi$ projects $B$ orthogonally onto a neighborhood of the origin in $T_{0}(\partial \Omega)$ (every $\bar{x} \in \partial \Omega$ has a neighborhood $B_{\bar{x}}$ which is diffeomorphic to a neighborhood of the origin in $T_{\bar{x}}(\partial \Omega)$ via orthogonal projection; pick a finite subcover and proceed as before).

For all $h \in T_{0}(\partial \Omega)$ with $h$ sufficiently small, $h$ is in the image of $\psi$ and one can define $k_{h}=\bar{x}(h)$. By Taylor's theorem

$$
k_{h}=\bar{x}(h)=\bar{x}(0)+(d \bar{x})_{0}(h)+\frac{1}{2} \sum_{i, j=1}^{n-1} \frac{\partial^{2} \bar{x}}{\partial u^{j} \partial u^{i}}(0) h^{i} h^{j}+o\left(|h|^{2}\right) .
$$

Since $\bar{x}(0)=0$ and $(d \bar{x})_{0}(h) \in T_{0}(\partial \Omega)$ it follows that

$$
k_{h} \cdot n_{0}=\frac{1}{2} L(h, h)+o(|h|)^{2} .
$$

On the other hand, Taylor's theorem and (2.7) give

$$
\phi\left(k_{h}\right)-d \phi_{0}\left(\left(k_{h}\right)_{t}\right)-\left(k_{h} \cdot n_{0}\right) \cdot \nu=\frac{1}{2} d^{2} \phi_{0}\left(k_{h}, k_{h}\right)+o\left(\left|k_{h}\right|^{2}\right) .
$$

Since $k_{h}=\bar{x}(h) \in \partial \Omega$ and $\left.\phi\right|_{\partial \Omega}=\psi$ the first two terms on the left side are orthogonal to $\nu$. Thus

$$
\begin{aligned}
-k_{n} \cdot n_{0} & =\frac{1}{2} d^{2} \phi_{0}\left(\left(k_{h}\right)_{t},\left(k_{h}\right)_{t}\right) \cdot \nu+o\left(\left|k_{h}\right|^{2}\right) \\
& =\frac{1}{2} d^{2} \phi_{0}(h, h) \cdot \nu+o\left(|h|^{2}\right)
\end{aligned}
$$

(since $\left(k_{h}\right)_{t}=h$, any $o\left(\left|k_{h}\right|^{2}\right)$ term is $\left.o\left(|h|^{2}\right)\right)$. Comparing this with (2.10) yields (2.9) and hence the result.

Proof of Lemma 2.3. For any real number $\delta$ define $I_{\delta}=\{x \in N: s \geqslant 0$ and, for all $\left.i, s-\varepsilon v_{i} \cdot n_{\bar{x}}-\varepsilon^{2} L\left(v_{i_{t}}, v_{i_{t}}\right) / 2 \geqslant \delta \varepsilon^{2}\right\}$. It is easy to show that for each $\delta>0$ there exists an $\varepsilon_{\delta}$ such that

$$
\varepsilon<\varepsilon_{\delta} \text { implies } I_{\delta} \subset S_{\delta} \subset I_{-\delta} .
$$

Next,

$$
\varepsilon<\varepsilon_{\delta} \text { implies }\left(S_{\varepsilon} \Delta I_{0}\right) \subset I_{-\delta} \backslash I_{\delta} .
$$

For, $x \in S_{\varepsilon} \Delta I_{0}$ if and only if $x \in S_{\varepsilon}$ but $x \notin I_{0}$ or $x \notin S_{\varepsilon}$ but $x \in I_{0}$. In the first case, $x \in S_{\varepsilon}$ implies $x \in I_{-\delta}$ by (2.11) and $x \notin I_{0}$ implies $x \notin I_{\delta}$. In the second case, $x \notin S_{\varepsilon}$ implies $x \notin I_{\delta}$ by (2.11) and $x \in I_{0}$ implies $x \in I_{-\delta}$. 
From (2.12) it follows that for $\varepsilon<\varepsilon_{\delta}$,

$$
\operatorname{vol}\left(S_{\varepsilon} \Delta I_{0}\right) \leqslant \operatorname{vol}\left(I_{-\delta} \backslash I_{\delta}\right)=\int_{\phi\left(I_{-\delta} \backslash I_{\delta}\right)}\left|d \phi^{-1}{ }_{(\bar{u}, s)}\right| d u^{1} \cdots d u^{n-1} d s .
$$

But

$$
\begin{aligned}
& \phi\left(I_{-\delta} \backslash I_{\delta}\right)=\{u \in \phi(N): s \geqslant 0 \text { and }- \delta \varepsilon^{2}+\max \left(\varepsilon v_{i} \cdot n_{\bar{x}}+\frac{\varepsilon^{2}}{2} L\left(v_{i_{t}}, v_{i_{t}}\right)\right) \\
&\left.\leqslant s<\delta \varepsilon+\max _{i}\left(\varepsilon v_{i} \cdot n_{\bar{x}}+\frac{\varepsilon^{2}}{2} L\left(v_{i_{t}}, v_{i_{t}}\right)\right)\right\} .
\end{aligned}
$$

Thus the above integral is

$$
\begin{aligned}
& \leqslant 2 \delta \varepsilon^{2} \sup _{\phi(N)}\left|d \phi_{(\cdot)}^{-1}\right| \int_{\phi\left(\partial \Omega \cap I_{-\delta} \backslash I_{\delta}\right)} d u^{1} \cdots d u^{n-1} \\
& \leqslant 2 \delta \varepsilon^{2} M,
\end{aligned}
$$

where $M=\sup _{\phi(N)}\left|d \phi_{(\cdot)}^{-1}\right| \operatorname{vol}_{\mathbf{R}^{n-1}}(\phi(B))$ (by the compactness of $\Omega$ one can guarantee this is finite). Thus it is established that for any $\delta>0$ there is an $\varepsilon_{\delta}$ such that for all $\varepsilon<\varepsilon_{\delta}, \operatorname{vol}\left(S_{\varepsilon} \Delta I_{0}\right) / \varepsilon^{2} \leqslant 2 \delta M$.

LEMMA 2.5. Let $\left\{v_{1}, \ldots, v_{r}\right\}$ be any finite set in $\mathbf{R}^{n}$ and define $\Omega_{v}=\Omega \cap\left(\Omega+v_{1}\right) \cap$ $\cdots \cap\left(\Omega+v_{r}\right)$. There exists a constant $C$, independent of the $v_{i}$ and of $r$, such that

$$
\left|\operatorname{vol}\left(\Omega \backslash \Omega_{v}\right)-\int_{\partial \Omega} \max _{i}\left(0, v_{i} \cdot n_{\bar{x}}\right) d A\right| \leqslant C \max _{i}\left(\left|v_{i}\right|^{2}\right) .
$$

Proof. For any positive number $p$ it can be assumed that $\max _{i}\left(\left|v_{i}\right|\right)<p$. For,

$$
\left|\operatorname{vol}\left(\Omega \backslash \Omega_{v}\right)-\int_{\partial \Omega} \max _{i}\left(0, v_{i} \cdot n_{\bar{x}}\right) d A\right| \leqslant \operatorname{vol}(\Omega)+\max _{i}\left(\left|v_{i}\right|\right) \operatorname{vol}(\partial \Omega)
$$

and if $\max \left(\left|v_{i}\right|\right) \geqslant p$ then the right side is less than $C_{1} \max \left(\left|v_{i}\right|\right)^{2}$ where

$$
C_{1}=\frac{\operatorname{vol}(\Omega)+p \operatorname{vol}(\partial \Omega)}{p^{2}} \text {. }
$$

Take $\max \left(\left|v_{i}\right|\right)$ small enough so that $\Omega \backslash \Omega_{v} \subset N_{0}$ and so that $x-v_{i} \in \operatorname{domain} \phi_{\tau}$ whenever $x \subset N_{\tau}$. Again using a partition of unity argument, it is enough to show

$$
\left|\int_{N_{\tau} \cap \Omega \backslash \Omega_{v}} \tilde{\rho}_{\tau} d V-\int_{B_{\tau}} \rho_{\tau}(\bar{x}) \max _{i}\left(0, v_{i} \cdot n_{\bar{x}}\right) d A\right| \leqslant C_{\tau} \max _{i}\left(\left|v_{i}\right|^{2}\right) .
$$

As before, the index $\tau$ will be dropped.

$x \in \Omega_{v} \cap N$ if and only if $s \geqslant 0$ and, for all $i, \phi\left(x-v_{i}\right) \cdot \nu \geqslant 0$. By Taylor's theorem $x \in \Omega_{v} \cap N$ if and only if $s \geqslant 0$ and, for all $i, \phi(x) \cdot \nu-d \phi_{x}\left(v_{i}\right) \cdot \nu+$ $R\left(v_{i}\right) \geqslant 0$ where $\left|R\left(v_{i}\right)\right| \leqslant M\left|v_{i}\right|^{2}$, the bound being uniform over $x$ since $d^{2} \phi$ is bounded on $N$. By Lemma 2.2, $x \in \Omega_{v} \cap N$ if and only if $s \geqslant 0$ and, for all $i$, $s-v_{i} \cdot n_{\bar{x}}+R\left(v_{i}\right) \geqslant 0$. 
Define $J_{M}=\left\{x \in N: s \geqslant 0\right.$ and, for all $\left.i, s-v_{i} \cdot n_{\bar{x}} \geqslant M\left(\max \left|v_{i}\right|^{2}\right)\right\}$. By an argument identical to that of Lemma 2.3 there is an $M_{1}$ such that $\operatorname{vol}\left(\left(\Omega_{v} \cap N\right) \Delta J_{0}\right)$ $\leqslant M_{1} \max \left(\left|v_{i}\right|^{2}\right)$ so $N \cap \Omega \backslash \Omega_{v}$ may be replaced by $N \cap \Omega \backslash J_{0}$.

By the mean value theorem (writing $u=\bar{u}+s$ )

$$
\begin{aligned}
& \int_{N \cap \Omega \backslash J_{0}} \tilde{\rho}_{\tau} d V=\int_{\phi\left(\Omega \cap N \backslash J_{0}\right)}\left(\tilde{\rho} \circ \phi^{-1}\right)\left|d \phi^{-1}{ }_{u}\right| d u^{1} \cdots d u^{n-1} d s \\
& \quad=\int_{\phi\left(\Omega \cap N \backslash J_{0}\right)}\left(\tilde{\rho} \circ \phi^{-1}\right)\left\{\left|d \phi^{-1}\right|+d\left(\left|d \phi^{-1}{ }_{(\cdot)}\right|\right)_{\bar{u}+s^{\prime} v}(s \nu)\right\} d u^{1} \cdots d u^{n-1} d s,
\end{aligned}
$$

where $0 \leqslant s^{\prime} \leqslant s$ and $\phi\left(\Omega \cap N \backslash J_{0}\right)=\left\{u \in \phi(N): 0 \leqslant s<\max _{i}\left(0, v_{i} \cdot n_{\bar{x}}\right)\right\}$. Performing the first integral on the right with respect to $s$ (keep in mind that $\tilde{\rho}$ is independent of $s$ ) and subtracting yields

$$
\begin{aligned}
& \left|\int_{N \cap \Omega \backslash J_{0}} \tilde{\rho} d V-\int_{B} \rho(\bar{x}) \max _{i}\left(0, v_{i} \cdot n_{\bar{x}}\right) d A\right| \\
& \quad=\left|\int_{\phi\left(\Omega \cap M \backslash J_{0}\right)} s\left(\tilde{\rho} \circ \phi^{-1}\right)\left(d\left|d \phi^{-1}(\cdot)\right|\right)_{\bar{u}+s^{\prime} \nu}(\nu) d u^{1} \cdots d u^{n-1} d s .\right|
\end{aligned}
$$

If $C_{1}=\sup \left\{|d| d \phi_{(\cdot)}^{-1}|| \cdot\left|\tilde{\rho} \circ \phi^{-1}\right|\right\}$ the last expression is

$$
\begin{aligned}
& \leqslant C_{1} \int_{\phi\left(\Omega \cap N \backslash J_{0}\right)} s d u^{1} \cdots d u^{n-1} d s \\
& =\frac{1}{2} C_{1} \int_{\phi(B)}\left(\max _{i}\left(0, v_{i} \cdot n_{\bar{x}}\right)\right)^{2} d u^{1} \cdots d u^{n-1} \\
& \leqslant \max \left(\left|v_{i}\right|^{2}\right) \cdot \frac{1}{2} C_{1} \int_{\phi(B)} d u^{1} \cdots d u^{n-1} .
\end{aligned}
$$

3. $\operatorname{tr}\left(T_{\alpha}^{m}\right)$ in the matrix case. In this section $k$ will be matrix valued. The notation $f * g$ will denote the convolution of $f$ and $g$ :

$$
f * g(x)=\int_{\mathbf{R}^{n}} f(x-y) g(y) d y .
$$

Note that (1.3) together with the assumption $\sigma \in L_{1}$ implies

(A) $\int|x||k(x)| d x<\infty$ which in turn implies $\int|x||k(x)|^{2} d x<\infty$, so the hypotheses of [7] are satisfied;

(B) $\int|x|^{2}|k(x)|^{2} d x<\infty$.

The following two lemmas will be needed.

LEMma 3.1. With $|k|^{(r)}$ denoting the $r$-fold convolution of $|k|$ with itself,

(i) $\int|x|\left(|k|^{(m-1)}(x)\right)^{2} d x \leqslant(m-1)^{2} \int|x||k(x)|^{2} d x\left\{\int|k(x)| d x\right\}^{2(m-2)}$;

(ii) the same estimate holds with $|x|$ replaced by $|x|^{2}$ on both sides. 
The proof is deferred to the end of the section. An easy consequence of Lemma 3.1 is

LEMMA 3.2. (i)

$$
\begin{gathered}
\int \cdots \int|x||k(x)|\left|k\left(x_{1}\right)\right| \cdots\left|k\left(x_{m-2}\right)\right|\left|k\left(-x-x_{1}-\cdots-x_{m-2}\right)\right| d x_{1} \cdots d x_{m-2} d x \\
\leqslant(m-1) \int|x||k(x)|^{2} d x\left\{\int|k(x)| d x\right\}^{m-2} .
\end{gathered}
$$

(ii) The same estimate holds with $|x|$ replaced by $|x|^{2}$ on both sides.

The statement of the main theorem of this section requires the following notation. The Hilbert transform (with respect to $\eta$ ) of an $L_{2}\left(T_{x}(\partial \Omega) \times \mathbf{R}\right.$ ) function $\sigma\left(\xi^{1}, \ldots, \xi^{n-1}, \eta\right)$ is

$$
\tilde{\boldsymbol{\sigma}}(\eta)=[i \operatorname{sgn}(t) \sigma(t)]^{\vee},
$$

the inverse Fourier transform being taken with respect to the last variable. It is convenient also to write

$$
\tilde{\sigma}(\eta)=\frac{1}{\pi} \mathrm{P} \cdot \mathrm{V} \cdot \int_{-\infty}^{\infty} \frac{\sigma\left(\xi^{1}, \ldots, \xi^{n-1}, \zeta\right)}{\zeta-\eta} d \zeta
$$

(the expression

$$
\frac{1}{\pi} \int_{|\zeta-\eta|>\varepsilon} \frac{\sigma\left(\xi^{1}, \ldots, \xi^{n-1}, \zeta\right)}{\zeta-\eta} d \zeta
$$

converges to $\tilde{\sigma}(\eta)$ in the $L_{2}$ norm as $\varepsilon$ tends to zero). At each $x \in \partial \Omega$ define

$$
\sigma_{ \pm}=\frac{1}{2}(\sigma \pm i \tilde{\sigma})
$$

and then, inductively,

$$
\begin{aligned}
& \Pi_{+}^{1}(\sigma)=\sigma_{+}, \quad \Pi_{-}^{1}(\sigma)=\sigma_{-}, \\
& \Pi_{+}^{n}(\sigma)=\left(\sigma \Pi_{+}^{n-1}(\sigma)\right)_{+}, \quad \Pi_{-}^{n}(\sigma)=\left(\Pi_{-}^{n-1}(\sigma) \cdot \sigma\right)_{-} .
\end{aligned}
$$

THEOREM 3.3. Under the assumptions outlined in the introduction

$$
\operatorname{tr}\left(T_{\alpha}^{m}\right)=a_{0} \alpha^{n}+a_{1} \alpha^{n-1}+a_{2} \alpha^{n-2}+o\left(\alpha^{n-2}\right), \quad \alpha \rightarrow \infty,
$$

where

$$
\begin{gathered}
a_{0}=\frac{1}{(2 \pi)^{n}} \operatorname{vol}(\Omega) \int \operatorname{tr} \sigma^{m}(\xi) d \xi \\
a_{1}=\frac{-i}{(2 \pi)^{n}} \sum_{q=1}^{m-1} \int_{T^{*}(\partial \Omega) \times \mathbf{R}} \operatorname{tr} \Pi_{+}^{q}(\sigma)^{n}(\xi) \Pi_{-}^{m-q}(\sigma)(\xi) d \xi d A
\end{gathered}
$$

and

$$
\begin{array}{r}
a_{2}=-\frac{1}{2(2 \pi)^{n}} \sum_{q=1}^{m-1} \int_{T^{*}(\partial \Omega) \times \mathbf{R}} \operatorname{tr}\left\{L\left(\operatorname{grad}_{\xi} \Pi_{+}^{q}(\sigma)(\xi), \operatorname{grad}_{\xi} \Pi_{-}^{m-q}(\sigma)(\xi)\right)\right. \\
\left.-\Pi_{+}^{q}(\sigma)^{n}(\xi) \Pi_{-}^{m-q}(\sigma)^{n}(\xi) \cdot H\right\} d \xi d A .
\end{array}
$$


Here $L\left(\operatorname{grad}_{\xi} \tau, \operatorname{grad}_{\xi} \tau\right)$ simply means $L_{i j} \tau^{i} \tau^{j}$ (summation convention). Also, $H$ has the same meaning as in Theorem 1.1.

Proof. By adding and subtracting

$$
\operatorname{tr} k\left(x_{1}\right) \cdots k\left(x_{m-1}\right) k\left(-x_{1}-\cdots-x_{m-1}\right) \operatorname{vol}(\alpha \Omega)
$$

to the integrand of (1.4) and using the fact that

$$
\begin{gathered}
\int \cdots \int k\left(x_{1}\right) \cdots k\left(x_{m-1}\right) k\left(-x_{1}-\cdots-x_{m-1}\right) d x_{1} \cdots d x_{m-1} \\
=k * \cdots * k(0)=\frac{1}{(2 \pi)^{n}} \int \sigma^{m}(\xi) d \xi
\end{gathered}
$$

(1.4) can be rewritten as

$$
\begin{aligned}
\operatorname{tr}\left(T_{\alpha}^{m}\right) & =\left(\frac{\alpha}{2 \pi}\right)^{n} \operatorname{vol}(\Omega) \int \operatorname{tr} \sigma^{m}(\xi) d \xi \\
-\int \cdots \int \operatorname{tr} k\left(x_{1}\right) \cdots k\left(x_{m-1}\right) k\left(-x_{1}-\cdots-x_{m-1}\right) & \\
\times & \operatorname{vol}(\alpha \Omega)-\operatorname{vol}\left(\alpha \Omega \cap \alpha \Omega+x_{1} \cap \cdots\right. \\
& \left.\left.\cap \alpha \Omega+x_{1}+\cdots+x_{m-1}\right)\right\} d x_{1} \cdots d x_{m-1} .
\end{aligned}
$$

By Lemma 2.5

$$
\begin{aligned}
& \alpha^{-n+2} \mid\left(\operatorname{vol}(\alpha \Omega)-\operatorname{vol}\left(\alpha \Omega \cap \alpha \Omega+x_{1} \cap \cdots \cap \alpha \Omega+x_{1}+\cdots+x_{m-1}\right)\right) \\
& \quad-\alpha^{n-1} \int_{\partial \Omega} \max \left(0, x_{1} \cdot n_{x}, \ldots,\left(x_{1}+\cdots+x_{m-1}\right) \cdot n_{x}\right) d A \mid \\
& \leqslant C \max _{i}\left(\left|x_{1}+\cdots+x_{1}\right|^{2}\right) \leqslant C(m-1)^{2} \sum_{i=1}^{m-1}\left|x_{i}\right|^{2} .
\end{aligned}
$$

Moreover, by Lemma 3.2,

$$
\begin{gathered}
\left.C(m-1)^{2} \int \cdots \int\left|k\left(x_{1}\right) \cdots k\left(x_{m-1}\right) k\left(-x_{1}-\cdots-x_{m-1}\right) \sum_{i=1}^{m-1}\right| x_{i}\right|^{2} \mid d x_{1} \cdots d x_{m-1} \\
\leqslant C(m-1)^{4} \int|x|^{2}|k(x)|^{2} d x\|k\|_{1}^{m-2} .
\end{gathered}
$$

Thus by the dominated convergence theorem

$$
\begin{gathered}
\lim _{\alpha \rightarrow \infty} \alpha^{-n+2} \int \cdots \int k\left(x_{1}\right) \cdots k\left(x_{m-1}\right) k\left(-x_{1}-\cdots-x_{m-1}\right) \\
\times\left\{\left(\operatorname{vol}(\alpha \Omega)-\operatorname{vol}\left(\alpha \Omega \cap \alpha \Omega+x_{1} \cap \cdots\right.\right.\right. \\
\left.\left.\cap \alpha \Omega+x_{1}+\cdots+x_{m-1}\right)\right) \\
\left.-\alpha^{n-1} \int_{\partial \Omega} \max \left(0, x_{1} \cdot n_{x}, \ldots,\left(x_{1}+\cdots+x_{m-1}\right) \cdot n_{x}\right) d A\right\} d x_{1} \cdots d x_{m-1}
\end{gathered}
$$


can be found by passing the limit under the integral. The result, by Corollary 2.4 , is

$$
\begin{gathered}
\frac{1}{2} \int \cdots \int \operatorname{tr} k\left(x_{1}\right) \cdots k\left(x_{m-1}\right) k\left(-x_{1}-\cdots-x_{m-1}\right) \\
\quad \times \sum_{q=1}^{m-1} \int_{\tilde{S}_{q}} L\left(x_{1_{t}}+\cdots+x_{q_{t}}, x_{1_{t}}+\cdots+x_{q_{t}}\right) \\
\quad-\left(\left(x_{1}+\cdots+x_{q}\right) \cdot n_{x}\right)^{2} H d A d x_{1} \cdots d x_{m-1},
\end{gathered}
$$

where $\tilde{S}_{q}=\left\{x \in \partial \Omega:\left(x_{1}+\cdots+x_{q}\right) \cdot n_{x}>\max _{i \neq q}\left(0,\left(x_{1}+\cdots+x_{i}\right) \cdot n_{x}\right)\right\}$.

Thus the expression in brackets in (3.2) can be replaced by

$$
\begin{gathered}
\alpha^{n-1} \int_{\partial \Omega} \max \left(0, x_{1} \cdot n_{x}, \ldots,\left(x_{1}+\cdots+x_{m-1}\right) \cdot n_{x}\right) d A \\
+\frac{\alpha^{n-2}}{2} \sum_{q=1}^{m-1} \int_{\tilde{S}_{q}} L\left(x_{1_{t}}+\cdots+x_{q_{t}}, x_{1_{t}}+\cdots+x_{q_{t}}\right) \\
\quad-\left(\left(x_{1}+\cdots+x_{q}\right) \cdot n_{x}\right)^{2} H d A
\end{gathered}
$$

with error $o\left(\alpha^{n-2}\right)$ and it follows that

$$
\begin{aligned}
a_{1}= & -\int \cdots \int \operatorname{tr} k\left(x_{1}\right) \cdots k\left(x_{m-1}\right) k\left(-x_{1}-\cdots-x_{m-1}\right) \\
& \times \int_{\partial \Omega} \max \left(0, x_{1} \cdot n_{x}, \ldots,\left(x_{1}+\cdots+x_{m-1}\right) \cdot n_{x}\right) d A d x_{1} \cdots d x_{m-1}, \\
a_{2}= & -\frac{1}{2} \int \cdots \int \operatorname{tr} k\left(x_{1}\right) \cdots k\left(x_{m-1}\right) k\left(-x_{1}-\cdots-x_{m-1}\right) \\
& \times \sum_{q=1}^{m-1} \int_{\tilde{S}_{q}} L\left(x_{1_{t}}+\cdots+x_{q_{t}}, x_{1_{1}}+\cdots+x_{q_{t}}\right) \\
& \quad-\left(\left(x_{1}+\cdots+x_{q}\right) \cdot n_{x}\right)^{2} H d A d x_{1} \cdots d x_{m-1} .
\end{aligned}
$$

Next, $\tilde{S}_{q}=\left\{x \in \partial \Omega\right.$ : for $s=1, \ldots, q, \sum_{r=s}^{q} x_{r} \cdot n_{x}>0$ and for $t=1, \ldots, m-1-q$, $\left.\sum_{r=q+1}^{q+t} x_{r} \cdot n_{x}<0\right\}$. Change variables: For $s=1, \ldots, q$ let $z_{s}=\sum_{r=s}^{q} x_{r}$ and for $t=1, \ldots, m-1-q$ let

$$
z_{q+t}=\sum_{r=q+1}^{q+t} x_{r}
$$

This gives

$$
\begin{aligned}
a_{1}= & -\sum_{q=1}^{m-1} \int_{\partial \Omega} d A \int \cdots \int z_{1} \cdot n_{x} \operatorname{tr} k\left(z_{1}-z_{2}\right) \cdots k\left(z_{q-1}-z_{q}\right) \\
& \times k\left(z_{q}\right) k\left(z_{q+1}\right) k\left(z_{q+2}-z_{q+1}\right) \cdots k\left(z_{m-1}-z_{m-2}\right) k\left(-z_{1}-z_{m-1}\right) \\
& \times \chi_{+}\left(z_{1} \cdot n_{x}\right) \cdots \chi_{+}\left(z_{q} \cdot n_{x}\right) \chi_{-}\left(z_{q+1} \cdot n_{x}\right) \cdots \chi_{-}\left(z_{m-1} \cdot n_{x}\right) d z_{1} \cdots d z_{m-1},
\end{aligned}
$$


where

$$
\chi_{+}\left(z \cdot n_{x}\right)= \begin{cases}1, & z \cdot n_{x} \geqslant 0 \\ 0, & z \cdot n_{x}<0\end{cases}
$$

and $\chi_{-}\left(z \cdot n_{x}\right)$ is defined in the obvious way. For $a_{2}$ the same expression is obtained except that $z_{1} \cdot n_{x}$ is replaced by $\frac{1}{2}\left\{L_{i j} z_{1_{t}}^{i} z_{1_{t}}^{j}-H\left(z_{1} \cdot n_{x}\right)^{2}\right\}$, where the $L_{i j}$ are the coefficients of the second fundamental form $L$ and $z_{1}^{i}$ is the $i$ th component of the projection of $z_{1}$ onto $T_{x} \partial \Omega$. The interchange in the order of integration in the $a_{2}$ term is justified by Fubini's theorem since the application of the dominated convergence theorem implied the integrand of the expression for $a_{2}$ in (3.4) is $L_{1}$. In the case of $a_{1}$, the justification follows easily from Lemma 3.2 .

Define

$$
k_{ \pm}(z)=k(z) \chi_{ \pm}\left(z \cdot n_{x}\right) .
$$

Note that if $\sigma_{ \pm}$is defined by (3.1) then

$$
k_{ \pm}(z)=\frac{1}{(2 \pi)^{n}} \int e^{i \xi \cdot z} \sigma_{ \pm}(\xi) d \xi
$$

Next, define inductively

$$
\begin{aligned}
& K_{+}^{1}(z)=k_{+}(z), \quad K_{-}^{1}(z)=k_{-}(z) \\
& K_{+}^{n}(z)=\left(k * K_{+}^{n-1}\right)_{+}(z), \quad K_{-}^{n}(z)=\left(K_{-}^{n-1} * k\right)_{-}(z) .
\end{aligned}
$$

Then

$$
\begin{aligned}
& a_{1}=-\sum_{q=1}^{m=1} \int_{\partial \Omega} \int z \cdot n_{x} \operatorname{tr} K_{+}^{q}(z) K_{-}^{m-q}(-z) d z d A, \\
& a_{2}=-\frac{1}{2} \sum_{q=1}^{m=1} \int_{\partial \Omega} \int\left\{L_{i j} z_{t}^{i} z_{t}^{j}-H\left(z \cdot n_{x}\right)^{2}\right\} \operatorname{tr} K_{+}^{q}(z) K_{-}^{m-q}(-z) d z d A .
\end{aligned}
$$

Now $z_{t}^{i} K_{+}^{q}(z)$ and $z_{t}^{j} K_{-}^{m-q}(-z)$ are in $L_{2}\left(\mathbf{R}^{n}\right)$ by Lemma 3.1. Moreover, using (3.6)

$$
\left(z_{t}^{j} K_{+}^{q}(z)\right)^{\wedge}=i \frac{\partial}{\partial \xi^{j}}\left(K_{+}^{q}\right)^{\wedge}=i \frac{\partial}{\partial \xi^{j}} \Pi_{+}^{q}(\sigma) .
$$

Similar formulas hold for the $z \cdot n_{x}$ terms. Altogether,

$$
\begin{aligned}
a_{1}=-i \sum_{q=1}^{m-1} \int_{\partial \Omega} \int \operatorname{tr}\left(\Pi_{+}^{q}(\sigma)^{n}\right)^{\vee}(z)\left(\Pi_{-}^{m-q}(\sigma)\right)^{\vee}(-z) d z d A, \\
a_{2}=-\frac{1}{2} \sum_{q=1}^{m-1} \int_{\partial \Omega} \int \operatorname{tr} L_{i j}\left(\Pi_{+}^{q}(\sigma)^{i}\right)^{\vee}(z)\left(\Pi_{-}^{m-q}(\sigma)^{j}\right)^{\vee}(-z) \\
-\left(\Pi_{+}^{q}(\sigma)^{n}\right)^{\vee}(z)\left(\Pi_{-}^{m-q}(\sigma)^{n}\right)^{\vee}(-z) \cdot H d z d A .
\end{aligned}
$$

The result is now immediate from the general formula

$$
\int k_{1}(z) k_{2}(-z) d z=\frac{1}{(2 \pi)^{n}} \int \hat{k}_{1}(\xi) \hat{k}_{2}(\xi) d \xi .
$$


Proof of Lemma 3.1. The proof of (i) is given, the proof of (ii) being essentially the same.

$$
\begin{aligned}
& \int|x|\left(|k|^{(m-1)}(x)\right)^{2} d x \\
& =\int\left\{\int \cdots \int\left|x-x_{1}+x_{1}-x_{2}+x_{2}-\cdots-x_{m-2}\right|^{1 / 2}\right. \\
& \left.\left|k\left(x-x_{1}\right)\right|\left|k\left(x-x_{2}\right)\right| \cdots\left|k\left(x_{m-2}\right)\right| d x_{1} \cdots d x_{m-2}\right\}^{2} d x \\
& \leqslant \int\left\{\int \cdots \int\left(\left|x-x_{1}\right|^{1 / 2}+\left|x_{1}-x_{2}\right|^{1 / 2}+\cdots+\left|x_{m-2}\right|^{1 / 2}\right)\right. \\
& \left.\left|k\left(x-x_{1}\right)\right|\left|k\left(x_{1}-x_{2}\right)\right| \cdots\left|k\left(x_{m-2}\right)\right| d x_{1} \cdots d x_{m-2}\right\}^{2} d x
\end{aligned}
$$

The terms in the integrand are all scalar valued and so commute. Thus the expression in brackets is the sum of $m-1$ terms, each of which is the convolution of $|x|^{1 / 2}|k(x)|$ and $m-2$ copies $|k(x)|$. That is, the above expression equals

$$
\begin{gathered}
\int\left\{(m-1) \int \cdots \int\left|x-x_{1}\right|^{1 / 2}\left|k\left(x-x_{1}\right)\right|\left|k\left(x_{1}-x_{2}\right)\right| \cdots\right. \\
\left.\left|k\left(x_{m-2}\right)\right| d x_{1} \cdots d x_{m-2}\right\}^{2} d x \\
=(m-1)^{2} \int \cdots \int\left\{\int\left|x-x_{1}\right|^{1 / 2}\left|k\left(x-x_{1}\right)\right|\left|x-z_{1}\right|^{1 / 2}\left|k\left(x-z_{1}\right)\right| d x\right\} \\
\times\left|k\left(x_{1}-x_{2}\right)\right| \cdots\left|k\left(x_{m-2}\right)\right|\left|k\left(z_{1}-z_{2}\right)\right| \\
\cdots\left|k\left(z_{m-2}\right)\right| d x_{1} \cdots d x_{m-2} d z_{1} \cdots d z_{m-2} .
\end{gathered}
$$

Since

$$
\begin{aligned}
\int \mid x & -\left.x_{1}\right|^{1 / 2}\left|k\left(x-x_{1}\right)\right|\left|x-z_{1}\right|^{1 / 2}\left|k\left(x-z_{1}\right)\right| d x \\
& \leqslant\left\{\int\left|x-x_{1}\right|\left|k\left(x-x_{1}\right)\right|^{2} d x\right\}^{1 / 2}\left\{\int\left|x-z_{1}\right|\left|k\left(x-z_{1}\right)\right|^{2} d x\right\}^{1 / 2}
\end{aligned}
$$

the last expression is less than or equal to

$$
(m-1)^{2} \int|x||k(x)|^{2} d x\left\{\int|k(x)| d x\right\}^{2(m-2)} .
$$

4. Proof of the main result. Throughout this section $k$ will be scalar valued. The following version of the identity of Spitzer will be used. Using the notation $k^{(n)}=k * \cdots * k$, where, as before, $*$ denotes convolution on $\mathbf{R}^{n}$, define

$$
E(k)-1=\sum_{n=1}^{\infty} \frac{k^{(n)}}{n !}
$$


and

$$
-L(1-f)=\sum_{n=1}^{\infty} \frac{k^{(n)}}{n} \text { for }\|k\|_{1}<1
$$

Then with $K_{+}^{n}(z)$ defined as in (3.7), the identity is

$$
\sum_{n=1}^{\infty} K_{+}^{n}(z)=E\left(-[L(1-k)]_{+}\right)-1,
$$

the plus on the right side being taken in the sense of (3.5).

LEMmA 4.1. If $|\lambda|>\|k\|_{1}$ and $L$ is defined as above then $L(1-k / \lambda) \in L_{1}\left(\mathbf{R}^{n}\right) \cap$ $L_{2}\left(\mathbf{R}^{n}\right)$.

Proof. If || || denotes either the $L_{1}$ or $L_{2}$ norm then

$$
\begin{aligned}
\left\|\sum_{n=1}^{\infty} \frac{k^{(n)}}{n|\lambda|^{n}}\right\| & \leqslant \sum_{n=1}^{\infty}\left\|\frac{k^{(n)}}{n|\lambda|^{n}}\right\| \leqslant \sum_{n=1}^{\infty} \frac{1}{n|\lambda|^{n}}\|k\|_{1}^{n-1}\|k\| \\
& \leqslant \frac{1}{|\lambda|}\|k\| \sum_{n=1}^{\infty} \frac{\|k\|_{1}^{n-1}}{|\lambda|^{n-1}} .
\end{aligned}
$$

LEMMA 4.2. (i) $\left\|\sigma^{\beta}\right\|_{2} \leqslant\|z k(z)\|_{2}$ for any $\beta=1, \ldots, n$.

(ii) $\left\|(\log (1-\sigma / \lambda))^{\beta}\right\|_{2} \leqslant(\inf |\lambda-\sigma|)^{-1}\left\|\sigma^{\beta}\right\|_{2}$.

Proof. (i) For instance, $\left\|\sigma^{i}\right\|_{2}=\left\|z_{t}^{i} k(z)\right\|_{2} \leqslant\|z k(z)\|_{2}$.

(ii) $(\log (1-\sigma / \lambda))^{\beta}=-\sigma^{\beta} /(\lambda-\sigma)$.

Since spec radius $\left(T_{\alpha}\right) \leqslant\left\|T_{\alpha}\right\| \leqslant\|k\|_{1}, F$ is analytic on $\operatorname{spec}\left(T_{\alpha}\right)$ for all $\alpha$. Let $\gamma(t) \subset \mathbf{C}$ be a circle of radius greater than $\|k\|_{1}$ centered at the origin and contained in the domain of analyticity of $F$. Then

$$
\left(\lambda-T_{\alpha}\right)^{-1}=\sum_{m=0}^{\infty} \frac{T_{\alpha}^{m}}{\lambda^{m+1}}
$$

Hence

$$
\begin{aligned}
\operatorname{tr} F\left(T_{\alpha}\right) & =\operatorname{tr} \frac{1}{2 \pi i} \int_{\gamma} F(\lambda)\left(\lambda-T_{\alpha}\right)^{-1} d \lambda \\
& =\operatorname{tr} \frac{1}{2 \pi i} \int_{\gamma} F(\lambda)\left\{\frac{1}{\lambda}+\frac{T_{\alpha}}{\lambda^{2}}+\sum_{m=2}^{\infty} \frac{T_{\alpha}^{m}}{\lambda^{m+1}}\right\} d \lambda
\end{aligned}
$$

Since $F(0)=0$, the first integral is zero. Also,

$$
\operatorname{tr} \frac{1}{2 \pi i} \int_{\gamma} F(\lambda) \frac{T_{\alpha}}{\lambda^{2}} d \lambda=\left(\frac{\alpha}{2 \pi}\right)^{n} \operatorname{vol}(\Omega) \frac{1}{2 \pi i} \int_{\gamma} \int F(\lambda) \frac{\sigma(\xi)}{\lambda^{2}} d \xi d \lambda
$$


since

$$
\operatorname{tr}\left(T_{\alpha}\right)=\left(\frac{\alpha}{2 \pi}\right)^{n} \operatorname{vol}(\Omega) \int \sigma(\xi) d \xi .
$$

Consider now the sum in (4.1). From $\S 3$

$$
\begin{aligned}
& \lim _{\alpha \rightarrow \infty} \alpha^{-n+2}\left\{\operatorname{tr}\left(T_{\alpha}^{m}\right)-\right.\left(\frac{\alpha}{2 \pi}\right)^{n} \operatorname{vol}(\Omega) \int \sigma^{m}(\xi) d \xi \\
&\left.+\alpha^{n-1} \sum_{q=1}^{m-1} \iint z \cdot n_{x} K_{+}^{q}(z) K_{-}^{m-q}(-z) d z d A\right\} \\
&=-\frac{1}{2} \sum_{q=1}^{m-1} \iint\left\{L_{i j} z_{t}^{i} z_{t}^{j}-\left(z \cdot n_{x}\right)^{2} H\right\} K_{+}^{q}(z) K_{-}^{m-q}(-z) d z d A .
\end{aligned}
$$

First using (3.2) and comparing the expression for $a_{1}$ in (3.4) and (3.8) and then using (3.3) gives, for all $\alpha \geqslant 1$,

$$
\begin{aligned}
& \alpha^{-n+2}\left|\operatorname{tr}\left(T_{\alpha}^{m}\right)-\left(\frac{\alpha}{2 \pi}\right)^{n} \operatorname{vol}(\Omega) \int \sigma^{m}(\xi) d \xi+\alpha^{n-1} \sum_{q=1}^{m-1} \iint z \cdot n_{x} K_{+}^{q}(z) K_{-}^{m-q}(-z) d z d A\right| \\
& =\alpha^{-n+2 \mid} \mid \int \cdots \int k\left(x_{1}\right) \cdots k\left(x_{m-1}\right) k\left(-x_{1}-\cdots-x_{m-1}\right) \\
& \times\left\{-\left(\operatorname{vol}(\alpha \Omega)-\operatorname{vol}\left(\alpha \Omega \cap \alpha \Omega+x_{1} \cap \cdots \cap \alpha \Omega+x_{1}+\cdots+x_{m-1}\right)\right)\right. \\
& \left.\quad+\alpha^{n-1} \int_{\partial \Omega} \max \left(0, x_{1} \cdot n_{x}, \ldots,\left(x_{1}+\cdots+x_{m-1}\right) \cdot n_{x}\right) d A\right\} d x_{1} \cdots d x_{m-1} \mid \\
& \leqslant C \int|x|^{2}|k(x)|^{2} d x(m-1)^{4}\|k\|_{1}^{m-2} .
\end{aligned}
$$

Since

$$
\int_{\gamma}|F(\lambda)| \sum_{m=2}^{\infty} \frac{1}{|\lambda|^{m+1}}(m-1)^{4}\|k\|_{1}^{m-2} d \lambda<\infty
$$

if $\|k\|_{1} /|\lambda|<1$ it follows from the above limit and the dominated convergence theorem that

$$
\begin{gathered}
\lim _{\alpha \rightarrow \infty} \alpha^{-n+2}\left[\frac { 1 } { 2 \pi i } \int _ { \gamma } F ( \lambda ) \sum _ { m = 2 } ^ { \infty } \frac { 1 } { \lambda ^ { m + 1 } } \left\{\operatorname{tr} T_{\alpha}^{m}-\left(\frac{\alpha}{2 \pi}\right)^{n} \operatorname{vol}(\Omega) \int \sigma^{m}(\xi) d \xi\right.\right. \\
\left.\left.+\alpha^{n-1} \sum_{q=1}^{m-1} \iint z \cdot n_{x} K_{+}^{q}(z) K_{-}^{m-q}(-z) d z d A\right\} d \lambda\right] \\
=-\frac{1}{2} \frac{1}{2 \pi i} \int_{\gamma} F(\lambda) \sum_{m=2}^{\infty} \frac{1}{\lambda^{m+1}} \iint\left\{L_{i j} z_{t}^{i} z_{t}^{j}-\left(z \cdot n_{x}\right)^{2} H\right\} \\
\times \sum_{q=1}^{m-1} K_{+}^{q}(z) K_{-}^{m-q}(-z) d z d A d \lambda
\end{gathered}
$$


From (4.1), (4.2) and (4.3)

$$
\begin{aligned}
\operatorname{tr} F\left(T_{\alpha}\right)= & \left(\frac{\alpha}{2 \pi}\right)^{n} \operatorname{vol}(\Omega) \frac{1}{2 \pi i} \iint F(\lambda) \sum_{m=1}^{\infty} \frac{\sigma^{m}(\xi)}{\lambda^{m+1}} d \xi d \lambda \\
& -\alpha^{n-1} \frac{1}{2 \pi i} \iiint F(\lambda) z \cdot n_{x} \sum_{m=2}^{\infty} \frac{1}{\lambda^{m+1}} \sum_{q=1}^{m-1} K_{+}^{q}(z) \\
& \times K_{-}^{m-q}(-z) d z d A d \lambda \\
- & \frac{\alpha^{n-2}}{2} \frac{1}{2 \pi i} \iiint F(\lambda)\left\{L_{i j} z_{t}^{i} z_{t}^{j}-\left(z \cdot n_{x}\right)^{2} H\right\} \\
& \times \sum_{m=2}^{\infty} \frac{1}{\lambda^{m+1}} \sum_{q=1}^{m-1} K_{+}^{q}(z) K_{-}^{m-q}(-z) d z d A d \lambda \\
& +o\left(\alpha^{n-2}\right), \quad \alpha \rightarrow \infty,
\end{aligned}
$$

where Lemma 3.2 is used to justify interchanging the infinite sums and integrals.

The first term on the right side is

$$
\left(\frac{\alpha}{2 \pi}\right)^{n} \operatorname{vol}(\Omega) \int F(\sigma(\xi)) d \xi
$$

The change in the order of integration is justified as follows:

$$
\sum_{m=1}^{\infty} \frac{\sigma^{m}}{\lambda^{m+1}}=\frac{1}{\lambda} \sum_{m=0}^{\infty} \frac{\sigma^{m}}{\lambda^{m}}-\frac{1}{\lambda}=\frac{1}{\lambda}\left(1-\frac{\sigma}{\lambda}\right)^{-1}-\frac{1}{\lambda}=\frac{\sigma}{\lambda(\lambda-\sigma)}
$$

and the $L_{1}$ norm of this is bounded by

$$
|\lambda|^{-1}(\inf |\lambda-\sigma|)^{-1}\|\sigma\|_{1}
$$

To handle the other terms note that

$$
\begin{aligned}
\sum_{m=2}^{\infty} & \frac{1}{\lambda^{m+1}} \sum_{q=1}^{m-1} K_{+}^{q}(z) K_{-}^{m-q}(-z) \\
& =\frac{1}{\lambda} \sum_{r=1}^{\infty} \frac{K_{+}^{r}(z)}{\lambda^{r}} \sum_{s=1}^{\infty} \frac{K_{-}^{s}(-z)}{\lambda^{s}} \\
& =\frac{1}{\lambda}\left(E\left(-\left[L\left(1-\frac{k}{\lambda}\right)\right]_{+}\right)(z)-1\right)\left(E\left(-\left[L\left(1-\frac{k}{\lambda}\right)\right]_{-}\right)(-z)-1\right)
\end{aligned}
$$

by Spitzer's identity. By Lemma 4.1 the expression $(-L(1-k / \lambda))_{ \pm}^{\wedge}$ makes sense and

$$
\left(E\left(-[L(1-k / \lambda)]_{ \pm}\right)-1\right)^{\wedge}=\exp (-\log (1-\hat{k} / \lambda))_{ \pm}-1=\tau^{ \pm}-1
$$

where the notation $\tau^{ \pm}$has been introduced for $\exp \left(\log (1-\sigma / \lambda)^{-1}\right)_{ \pm}$.

Using (4.4), (4.6), and (4.7) and then integrating by parts twice with respect to $\eta$ 
yields

$$
\begin{aligned}
a_{1} & =-\frac{1}{2 \pi i} \iiint \frac{F(\lambda)}{\lambda} z \cdot n_{x}\left(\tau^{+}-1\right)^{\vee}(z)\left(\tau^{-}-1\right)^{\vee}(-z) d z d A d \lambda \\
& =-\frac{1}{2 \pi i} \frac{1}{(2 \pi)^{n}} \iiint \frac{F(\lambda)}{\lambda} i\left(\tau^{+}\right)^{n}(\xi)\left(\tau^{-}-1\right)(\xi) d \xi d A d \lambda \\
& =-\frac{1}{2 \pi i} \frac{1}{(2 \pi)^{n}} \iiint \frac{F(\lambda)}{\lambda} i \tau^{+}(\xi)\left(\tau^{-}\right)^{n}(\xi) d \xi d A d \lambda \\
& =-\frac{1}{2 \pi i} \frac{1}{(2 \pi)^{n}} \iiint \frac{F(\lambda)}{\lambda} i\left(\tau^{+}\right)^{n}(\xi) \tau^{-}(\xi) d \xi d A d \lambda .
\end{aligned}
$$

For any $\beta=1, \ldots, n$

$$
\left(\tau^{ \pm}\right)^{\beta}=\tau^{ \pm}\left(\log (1-\sigma / \lambda)^{-1}\right)_{ \pm}^{\beta} .
$$

By Lemma 4.2 the expression $(-\log (1-\sigma / \lambda))_{ \pm}^{\beta}$ makes sense and one has almost everywhere (using the definition of )

$$
\left(\log (1-\sigma / \lambda)^{-1}\right)_{ \pm}^{\beta}=\left(\sigma^{\beta} /(\lambda-\sigma)\right)_{ \pm} .
$$

Also,

$$
\tau^{+} \tau^{-}=\lambda(\lambda-\sigma)^{-1}
$$

Thus, (4.8) can be rewritten as

$$
a_{1}=\frac{1}{(2 \pi)^{n}} \frac{1}{2 \pi i} \iiint F(\lambda) i(\lambda-\sigma)^{-1}\left(\frac{\sigma^{n}}{\lambda-\sigma}\right)_{-} d \xi d A d \lambda
$$

and (4.9) can be written as

$$
a_{1}=-\frac{1}{(2 \pi)^{n}} \frac{1}{2 \pi i} \iiint F(\lambda) i(\lambda-\sigma)^{-1}\left(\frac{\sigma^{n}}{\lambda-\sigma}\right)_{+} d \xi d A d \lambda .
$$

Since in general $\sigma_{-}-\sigma_{+}=-i \tilde{\sigma}$ these two expressions can be added to give

$$
\begin{aligned}
a_{1} & =\frac{1}{2} \frac{1}{(2 \pi)^{n}} \frac{1}{2 \pi i} \iiint F(\lambda)(\lambda-\sigma)^{-1}\left(\frac{\sigma^{n}}{\lambda-\sigma}\right) d \xi d A d \lambda \\
& =\frac{1}{2} \frac{1}{(2 \pi)^{n}} \frac{1}{2 \pi i} \iiint \frac{1}{\pi} \int F(\lambda)(\lambda-\sigma(\eta))^{-1} \frac{\sigma^{n}(\zeta)}{\lambda-\sigma(\zeta)} \frac{d \zeta}{\zeta-\eta} d \xi d A d \lambda \\
& =\frac{1}{4 \pi} \frac{1}{(2 \pi)^{n}} \iiint \frac{F(\sigma(\eta))-F(\sigma(\zeta))}{\sigma(\eta)-\sigma(\zeta)} \frac{\sigma^{n}(\zeta)-\sigma^{n}(\eta)}{\zeta-\eta} d \zeta d \xi d A
\end{aligned}
$$

The interchange in the order of integration is justified as follows.

$$
\int\left(\frac{\sigma^{n}}{\lambda-\sigma}\right)^{\tilde{d}} d \xi=\int\left(-\log \left(1-\frac{\sigma}{\lambda}\right)\right)^{n \sim} d \xi=\int\left(-\log \left(1-\frac{\sigma}{\lambda}\right)\right)^{n} d \xi=0 .
$$


Thus

$$
\begin{aligned}
\int(\lambda-\sigma)^{-1}\left(\frac{\sigma^{n}}{\lambda-\sigma}\right)^{\tilde{d}} d \xi & =\int\left\{(\lambda-\sigma)^{-1}-\frac{1}{\lambda}\right\}\left(\frac{\sigma^{n}}{\lambda-\sigma}\right)^{\tilde{\lambda}} d \xi \\
& =\int \frac{1}{\lambda} \frac{\sigma}{\lambda-\sigma}\left(\frac{\sigma^{n}}{\lambda-\sigma}\right)^{\tilde{d}} d \xi
\end{aligned}
$$

and using Lemma 4.2 and the fact that the Hilbert transform is norm preserving

$$
\begin{aligned}
\iiint & \left|\frac{F(\lambda)}{\lambda} \frac{\sigma}{\lambda-\sigma}\left(\frac{\sigma^{n}}{\lambda-\sigma}\right)^{\tilde{l}}\right| d \xi d A d \lambda \\
& \leqslant \int_{\gamma} \int_{\partial \Omega} \frac{|F(\lambda)|}{|\lambda|}\left\|\frac{\sigma}{\lambda-\sigma}\right\|_{2}\left\|\frac{\sigma^{n}}{\lambda-\sigma}\right\|_{2} d A d \lambda \\
& \leqslant|\lambda|^{-1}(\inf |\lambda-\sigma|)^{-2}\|\sigma\|_{2}\|z k(z)\|_{2} \operatorname{vol}(\partial \Omega) \int_{\gamma}|F(\lambda)| d \lambda \\
& <\infty .
\end{aligned}
$$

Thus $d \lambda$ may be interchanged with $d \xi d A$. To interchange $d \lambda$ with $d \zeta$ note that for fixed $\eta$ the change of variable $\bar{\zeta}=\zeta-\eta$ gives

$$
\begin{aligned}
\lim _{\varepsilon \rightarrow 0} \int_{|\zeta-\eta|>\varepsilon} \frac{\sigma^{n}(\zeta)}{\lambda-\sigma(\zeta)} \frac{1}{\zeta-\eta} d \zeta & =\lim _{\varepsilon \rightarrow 0} \int_{|\zeta|>\varepsilon} \frac{\sigma^{n}(\zeta+\eta)}{\lambda-\sigma(\zeta+\eta)} \frac{1}{\zeta} d \zeta \\
& =\lim _{\varepsilon \rightarrow 0} \int_{\varepsilon}^{\infty}\left\{\frac{\sigma^{n}(\eta+\zeta)}{\lambda-\sigma(\eta+\zeta)}-\frac{\sigma^{n}(\eta-\zeta)}{\lambda-\sigma(\eta-\zeta)}\right\} \frac{1}{\zeta} d \zeta
\end{aligned}
$$

The integrand in the last expression is continuous for $\zeta \neq 0$ and

$$
\lim _{\zeta \rightarrow 0^{+}} \frac{1}{\zeta}\left\{\frac{\sigma^{n}(\eta+\zeta)}{\lambda-\sigma(\eta+\zeta)}-\frac{\sigma^{n}(\eta-\zeta)}{\lambda-\sigma(\eta-\zeta)}\right\}=0
$$

since $\sigma$ is twice differentiable. Hence the integration with respect to $\zeta$ is actually proper at $\zeta=0$ and the integrand is $L_{1}$ if the domain of integration is restricted to $0 \leqslant \zeta \leqslant 1$. On the other hand, for $1<\zeta<\infty$

$$
\begin{aligned}
\int_{\gamma} \int_{1}^{\infty} & F(\lambda)(\lambda-\sigma(\eta))^{-1} \frac{\sigma^{n}(\eta+\zeta)}{\lambda-\sigma(\eta+\zeta)} \frac{1}{\zeta} \mid d \zeta d \lambda \\
& \leqslant\left\|\sigma^{n}\right\|_{2}\left\|\frac{1}{\zeta}\right\|_{2}(\inf |\lambda-\sigma|)^{-2} \int_{\gamma}|F(\lambda)| d \lambda<\infty .
\end{aligned}
$$

Now for $a_{2}$. From (4.4), (4.6) and (4.7)

$$
\begin{array}{r}
a_{2}=-\frac{1}{2} \frac{1}{2 \pi i} \iiint \frac{F(\lambda)}{\lambda}\left[L_{i j} z_{t}^{i} z_{t}^{j}-\left(z \cdot n_{x}\right)^{2} H\right] \\
\quad \times\left(\tau^{+}-1\right)^{\vee}(z)\left(\tau^{-}-1\right)^{\vee}(-z) d z d A d \lambda \\
=-\frac{1}{2} \frac{1}{(2 \pi)^{n}} \frac{1}{2 \pi i} \iiint \frac{F(\lambda)}{\lambda}\left\{L_{i j}\left(\tau^{+}\right)^{i}(\xi)\left(\tau^{-}\right)^{j}(\xi)\right. \\
\left.-H\left(\tau^{+}\right)^{n}(\xi)\left(\tau^{-}\right)^{n}(\xi)\right\} d \xi d A d \lambda .
\end{array}
$$


From (4.10), (4.11) and (4.12) this equals

$$
\begin{aligned}
a_{2}=-\frac{1}{2} \frac{1}{(2 \pi)^{n}} \frac{1}{2 \pi i} \iiint \frac{F(\lambda)}{\lambda-\sigma(\xi)}\{ & L_{i j}\left(\frac{\sigma^{i}}{\lambda-\sigma}\right)_{+}(\xi)\left(\frac{\sigma^{j}}{\lambda-\sigma}\right)_{-}(\xi) \\
& \left.-H\left(\frac{\sigma^{n}}{\lambda-\sigma}\right)_{+}(\xi)\left(\frac{\sigma^{n}}{\lambda-\sigma}\right)_{-}(\xi)\right\} d \xi d A d \lambda .
\end{aligned}
$$

Writing out the Hilbert transforms explicitly and then performing a straightforward residue calculation yields

$$
\begin{array}{r}
a_{2}=-\frac{1}{16} \frac{1}{(2 \pi)^{n}} \iint F^{\prime \prime}(\sigma(\xi))\left\{L_{i j} \sigma^{i}(\xi) \sigma^{j}(\xi)-H\left(\sigma^{n}(\xi)\right)^{2}\right\} d \xi d A \\
-\frac{1}{8 \pi^{2}} \frac{1}{(2 \pi)^{n}} \iiint \int\left\{\frac{F(\sigma(\eta))}{\left(\sigma(\eta)-\sigma\left(\zeta_{1}\right)\right)\left(\sigma(\eta)-\left(\zeta_{2}\right)\right)}\right. \\
+\frac{F\left(\sigma\left(\zeta_{1}\right)\right)}{\left(\sigma\left(\zeta_{1}\right)-\sigma(\eta)\right)\left(\sigma\left(\zeta_{1}\right)-\sigma\left(\zeta_{2}\right)\right)} \\
\left.\quad+\frac{F\left(\sigma\left(\zeta_{2}\right)\right)}{\left(\sigma\left(\zeta_{2}\right)-\sigma(\eta)\right)\left(\sigma\left(\zeta_{2}\right)-\sigma\left(\zeta_{1}\right)\right)}\right\} \\
\times\left(L_{i j} \sigma^{i}\left(\zeta_{1}\right) \sigma^{j}\left(\zeta_{2}\right)-H \sigma^{n}\left(\zeta_{1}\right) \sigma^{n}\left(\zeta_{2}\right)\right) \frac{d \zeta_{1}}{\zeta_{1}-\eta} \frac{d \zeta_{2}}{\zeta_{2}-\eta} d \xi d A .
\end{array}
$$

It will be shown that the first term in the above expression is zero. The theorem then follows from (4.4), (4.5), (4.14) and (4.15). It is enough to show the term is zero in the case $F$ is a power, $F(\lambda)=\lambda^{m}$, since in the general case $F^{\prime \prime}$ is analytic on the range of $\sigma$. For $F(\lambda)=\lambda^{m}$ the expression in question is

$$
\begin{aligned}
-\frac{1}{16} & \frac{1}{(2 \pi)^{n}} \int_{\partial \Omega} \int m(m-1)(\sigma(\xi))^{m-2}\left\{L_{i j} \sigma^{i} \sigma^{j}-H\left(\sigma^{n}\right)^{2}\right\} d \xi d A \\
& =-\frac{1}{16} \frac{1}{(2 \pi)^{n}} \int_{\partial \Omega} \int m\left\{L_{i j}\left(\sigma^{m-1}\right)^{i} \sigma^{j}-H\left(\sigma^{m-1}\right)^{n} \sigma^{n}\right\} d \xi d A \\
& =-\frac{m}{16} \int_{\partial \Omega} \int\left\{L_{i j} z_{t}^{i} z_{t}^{j}-\left(z \cdot n_{x}\right)^{2} H\right\} k^{(m-1)}(z) k(-z) d z d A .
\end{aligned}
$$

It is easy to justify interchanging the order of integration and the result then follows from an identity discovered by Widom and J. Dadok:

$$
\int_{\partial \Omega} L\left(z_{t}, z_{t}\right)-\left(z \cdot n_{x}\right)^{2} H d A=0 .
$$

The following proof is the result of communications with R. Osserman, L. Simon, and B. Lawson. It is known that if $X$ is a vector field along $M$, a closed smooth hypersurface in $\mathbf{R}^{n}$, then

$$
\int_{M} \sum_{i=1}^{n-1}\left\langle\nabla_{\tau_{i}} X, \tau_{i}\right\rangle d A(x)=\int_{M}\left\langle X, n_{x}\right\rangle H d A(x),
$$


where the $\tau_{i}$ are an orthonormal basis for the tangent space to $M$ at $x, \nabla_{v}$ denotes covariant differentiation in the direction of $v$, and the brackets denote the ordinary $\mathbf{R}^{n}$ inner product. To prove this note

$$
\sum_{i}\left\langle\nabla_{\tau_{i}} X, \tau_{i}\right\rangle=\sum_{i}\left\langle\nabla_{\tau_{i}}\left\langle X, n_{x}\right\rangle n_{x}, \tau_{i}\right\rangle+\sum_{i}\left\langle\nabla_{\tau_{i}} X_{t}, \tau_{i}\right\rangle
$$

The integral over $M$ of the second term is zero by the divergence theorem (see [4, pp. 188 and 193]) and the first term equals

$$
\left\langle X, n_{x}\right\rangle \sum_{i}\left\langle\nabla_{\tau_{i}} n_{x}, \tau_{i}\right\rangle=\left\langle X, n_{x}\right\rangle H .
$$

To prove (4.16) take $X=\left\langle z, n_{x}\right\rangle z$ in (4.17). The right side immediately gives (with $M=\partial \Omega$ )

$$
\int_{\partial \Omega}\left\langle z, n_{x}\right\rangle^{2} H d A
$$

The left side gives

$$
\int_{\partial \Omega} \sum_{i}\left\langle z, \nabla_{\tau_{i}} n_{x}\right\rangle\left\langle z, \tau_{i}\right\rangle d A
$$

Since $\nabla_{\tau_{i}} n_{x}=W_{n_{x}}\left(\tau_{i}\right)$, where $W_{n_{x}}$ is the Weingarten map and the latter is selfadjoint, the integrand of this expression equals

$$
\sum_{i}\left\langle W_{n_{x}}\left(z_{t}\right), \tau_{i}\right\rangle\left\langle z, \tau_{i}\right\rangle=\left\langle W_{n_{x}}\left(z_{t}\right), z_{t}\right\rangle=L\left(z_{t}, z_{t}\right) .
$$

5. The logarithm. Let $F(\lambda)=\log (1+\lambda)$ and let $\gamma$ be the circle of radius $r$ about the origin with $\|k\|_{1}<r<1$. The following method of deriving the expression for $a_{1}$ in (1.2) from the expression in the main result was shown to the author by Widom. Justification of the various steps is accomplished as in $§ 4$.

From (4.14) and the first line of (4.13)

$$
\begin{aligned}
a_{1} & =\frac{1}{2} \frac{1}{(2 \pi)^{n}} \frac{1}{2 \pi i} \int_{\gamma} \int_{\partial \Omega} \int \log (1+\lambda) \frac{\sigma}{\lambda(\lambda-\sigma)}\left(\frac{\sigma^{n}}{\lambda-\sigma}\right)^{\tilde{\lambda}} d \xi d A d \lambda \\
& =\frac{1}{2} \frac{1}{2 \pi i} \int_{\gamma} \int_{\partial \Omega} \int \log (1+\lambda)\left|z \cdot n_{x}\right| s_{\lambda}^{\prime}(z) s_{\lambda}(-z) d z d A d \lambda,
\end{aligned}
$$

where $s_{\lambda}(z)=(\log (1-\sigma / \lambda))^{\vee}(z)$ and the prime denotes differentiation with respect to $\lambda$. Since $\left|z \cdot n_{x}\right|$ is invariant under changing $z$ to $-z$ it follows that

$$
\begin{aligned}
a_{1} & =\frac{1}{4} \frac{1}{2 \pi i} \int_{\gamma} \int_{\partial \Omega} \int \log (1+\lambda)\left|z \cdot n_{x}\right| \frac{d}{d \lambda}\left(s_{\lambda}(z) s_{\lambda}(-z)\right) d z d A d \lambda \\
& =-\frac{1}{4} \frac{1}{2 \pi i} \int_{\gamma} \int_{\partial \Omega} \int \frac{1}{\lambda+1}\left|z \cdot n_{x}\right| s_{\lambda}(z) s_{\lambda}(-z) d z d A d \lambda .
\end{aligned}
$$

Let $\tilde{\gamma}$ be a circle centered at -1 with radius less than $1-r$. Then

$$
a_{1}=\frac{1}{4} \frac{1}{2 \pi i} \int_{\tilde{\gamma}} \int_{\partial \Omega} \int \frac{1}{\lambda+1}\left|z \cdot n_{x}\right| s_{\lambda}(z) s_{\lambda}(-z) d z d A d \lambda
$$

and performing the integration with respect to $\lambda$ gives the result. 


\section{REFERENCES}

1. H. Dym and H. P. McKean, Fourier series and integrals, Academic Press, New York, 1972.

2. U. Grenander and G. Szegö, Toeplitz forms and their applications, Univ. of California Press, 1968.

3. M. Kac, Toeplitz matrices, translation kernels, and a related problem in probability theory, Duke Math J. 21 (1954), 501-509.

4. M. Spivak, A comprehensive introduction to differential geometry, vol. 4, Publish or Perish, Berkeley, Calif., 1979.

5. J. Thorpe, Elementary topics in differential geometry, Springer-Verlag, New York, 1979.

6. H. Widom, $A$ theorem on translation kernels in $n$ dimensions, Trans. Amer. Math. Soc. 94 (1960), 170-180.

7. $182-198$.

Szegö's limit theorem: the higher dimensional matrix case, J. Funct. Anal. 39 (1980),

8.

Asymptotic expansions of determinants for families of trace class operators: Corrigendum and Addendum, Indiana Univ. Math. J. 33 (1984), 277-288.

Department of Mathematics, San Jose State University, San Jose, California 95192 